\title{
Proteome-wide identification of lysine succinylation in the proteins of Eucommia ulmoides Oliver leaves revealed its
} involvement in energy metabolism

\author{
Chao Shen ${ }^{1}$, Xinzhuan Yao ${ }^{1}$, Degang Zhao ${ }^{2}$ and Litang $\mathrm{Lu}^{1,2}$ \\ ${ }^{1}$ College of Tea Science, Guizhou University, Guiyang, Guizhou, People's Republic of China \\ 2 The Key Laboratory of Plant Resources Conservation and Germplasm Innovation in Mountainous Region (Ministry of \\ Education), Institute of Agro-Bioengineering, Guiyang, Guizhou, People's Republic of China
}

\begin{abstract}
Summary
The lysine succinylation, evolutionarily conserved post-translational modification (PTM), played an important regulatory role in physiology of both eukaryotes and prokaryotes. Large succinyl groups conjugated to lysine residues resulted in changes in structure and charge of the proteins and affected the function of these proteins. However, little information was available about the lysine succinylation in the Eucommia ulmoides, an important traditional Chinese herb with multiple active substances. In this study, we performed lysine succinylome analysis to determine the potential regulatory role of lysine succinylation in growth and development of $E$. ulmoides by LC-MS/ MS combined with immune-purification. A total of 653 lysine succinylation sites distributed in 365 proteins were identified. Gene ontology (G0) analysis showed that lysine-succinylated proteins are related to a wide range of cellular functions, from metabolic processes to stimuli responses. Bioinformatic analysis showed that succinylated proteins were mainly distributed in chloroplast (176 proteins), cytoplasm ( 99 proteins) and mitochondria (33 proteins) which are mainly involved in energy metabolism, including photosynthesis, Calvin-Benson cycle, glycolysis and TCA cycle. Our research suggested that lysine succinylation was necessary for life activities, which were mainly involved in the process of converting solar energy into biomass energy stored and converting the energy stored by plants into energy (ATP) available for direct use in E. ulmoides. This finding provided a research basis for further revealing the mechanism of lysine succinylation regulating various physiological activities in plants.
\end{abstract}

Keywords

Calvin-Benson cycle, Eucommia ulmoides Oliver,

glycolysis, lysine succinylation, photosynthesis, TCA cycle

\section{Introduction}

Eucommia ulmoides Oliver is a deciduous tree belonging to the monotypic family Eucommiaceae, which was considered to have high medicinal and economic values due to a lot of compounds and high-quality rubber in the world. As a traditional Chinese medicine (TCM) plant, a total of 112 compounds had been detected in the leaves and barks of

\section{Significance of this study}

What is already known on this subject?

- Protein post-translational modifications (PTMs) affect the spatial conformation, stability, activity, and protein interaction. Previous studies revealed that PTMs were mainly involved in various cellular and metabolic processes, including metabolic pathways, cell differentiation, cell cycle regulation, apoptosis, etc. Lysine succinylation (Ksuc) modification is relatively inadequate in comparison with acetylation in plants; reports on lysine succinylation modification mainly focus on animals, especially in the vital organs of mammals.

What are the new findings?

- In this study, we firstly performed lysine succinylome analysis to determine the potential regulatory role of lysine succinylation in growth and development of Eucommia ulmoides by LC-MS/MS combined with immune-purification. Our research suggested that lysine succinylation was necessary for life activities involved in the process of converting solar energy into biomass energy stored and converting the energy stored by plants into energy (ATP) available for use in E. ulmoides.

What is the expected impact on horticulture?

- We deciphered the role of protein succinylation in E. ulmoides, and made a prediction of its role in E. ulmoides. Lysine succinate modification may play an important role in providing energy for the growth and development of plants. The dataset can be used to examine the functions of lysine succinylation and provided a research basis for revealing the mechanism of lysine succinylation regulating physiological activities in plants.

E. ulmoides, which were roughly divided into the following categories: lignans, iridoids, phenylpropanoids, flavonoids, and polysaccharide. These compounds have pharmacological effects on pain relief, anti-hypertension, debasing fattiness, controlling coronary blood flow, diuresis and diabetes (Dai et al., 2013; Horii et al., 2010; Park et al., 2006; Zhu et al., 2016). Eucommia rubber is known as gutta-percha, having the chemical structure of trans-polyisoprene $\left(\mathrm{C}_{5} \mathrm{H}_{8}\right)$ n, which was richly distributed in E. ulmoides. Eucommia rubber, as a 
common natural rubber Brazilian rubber (cis-polyisoprene) isomers, which has excellent insulation properties, acid and alkali resistance, high rigidity, and so on (Bamba et al., 2002; Chen et al., 2008). In addition, the publication and release of the $E$. ulmoides genome provides an opportunity to study genome-wide protein modifications (Wuyun et al., 2018).

Protein post-translational modifications (PTMs), a dynamic switch and reversible covalent process, which affected the spatial conformation, stability, activity, and protein interaction due to change the physical and chemical properties of proteins by adding/removing modification groups to/from amino acid residue(s) (Walsh et al., 2005; Witze et al., 2007). More than 400 types of PTMs, including methylation, acetylation, phosphorylation and ubiquitination, had been discovered (Wani et al., 2015; Choudhary et al., 2009). Previous studies had revealed that PTMs were mainly involved in various cellular and metabolic processes, including metabolic pathways, cell differentiation, cell cycle regulation and apoptosis, etc. (Cantin et al., 2004). Since Rosen et al. (2004) firstly identified lysine succinylation (Ksuc), revealed that Ksuc was involved in the first step of methionine synthesis in E. coli. The recent studies had revealed that the lysine succinylation was widely distributed in different species (Weinert et al., 2013), for example microorganisms (Candida albicans, Aspergillus flavus) (Zheng et al., 2016; Ren et al., 2018), animals (Rattus norvegicus, Homo sapiens) (Cheng et al., 2016; Song et al., 2017), and plants (Dendrobium officinale, Camellia sinensis and Oryza sativa) (Feng et al., 2017; Xu et al., 2017; Meng et al., 2019).

Lysine succinylation (Ksuc) modification, defined as a transfer of a succinyl group to a lysine residue of a protein, is a newly identified protein post-translational modification (Papanicolaou et al., 2014). Compared with methylation and acetylation, lysine succinylation can trigger more changes in protein properties. Due to the succinyl group bringing two negative charges to the lysine residue causing the valence state changes from +1 to -1 , which is higher than acetylation ( +1 to 0 ) and monomethylation (no change). In addition, the lysine carries a larger structure group after lysine succinylation modification to further affect the physiological and biochemical properties, and protein functions (Zhang et al., 2010). Recent studies had revealed that lysine succinylation and acetylation have a high degree of overlap, indicating that there may be potential interactions between the two in the organism to complete the regulation of complex life activities. The study of lysine succinylation is relatively inadequate in comparison with acetylation in plants. Reports on lysine succinylation modification mainly focus on animals, especially in the vital organs of mammals. For example, a total of 2,004 modified sites were found in human cervical cancer cells, and 2,140 modified sites were found in mouse liver tissue (Weinert et al., 2013).

Recent studies also found that lysine succinylation modification was distributed in some plants. The analysis of succinylome in monocot was performed and found 330 lysine succinylation modification sites (173 proteins) in wheat (Zhang et al., 2017) and 665 lysine succinylation modification sites (261 proteins) in rice seed (He et al., 2016). In addition, the lysine succinylation modification was explored in some dicots, such as strawberry (Fang et al., 2018), tomato (Jin et al., 2016), and tea (Xu et al., 2017). The lysine succinylation modification in Chinese herbal medicine plants was also detected, including Dendrobium officinale (Feng et al., 2017), Taxus $\times$ media (Shen et al., 2016) and Patchouli plant (Wang et al., 2019). Interestingly, these studies had revealed that the succinylation modification of lysine was involved in various metabolic pathways, especially tricarboxylic acid (TCA) cycle, glycolysis, and carbohydrate metabolism (Colak et al., 2013). Even with the above studies on lysine succinylation modification in plants, it is still insufficient compared with other modifications and the study of this modification in mammals and microorganisms, and needs further research.

Although lysine succinylation plays an important role in the regulation of various life activities, there is no report on the succinylation of lysine in E. ulmoides. So systematic identification of the lysine succinylome of $E$. ulmoides was done using affinity enrichment technique combined with LC-MS/ MS in this study. Then, using bioinformatics analysis to further explore the potential regulatory effects of lysine succinylation. So far, this is the first report on the succinylation of lysine in E. ulmoides, and it will also provide a research basis for further revealing the regulatory mechanism of succinylation of lysine in plant life activities.

\section{Materials and methods}

\section{Plant material collection and protein extraction}

Leaf samples of E. ulmoides were collected from the farm of Guizhou University and frozen immediately in liquid at $\mathrm{N}_{2}$, stored at $-80^{\circ} \mathrm{C}$ refrigerator. The protein was extracted by referring to the method of Sadhukhan et al. (2016). All samples were first grinded into powder by liquid nitrogen and transferred to 5-mL centrifuge tube and sonicated three times on ice in lysis buffer (including 1\% TritonX-100, 10 mM dithiothreitol, and 1\% Protease Inhibitor Cocktail, $50 \mu \mathrm{M}$ PR-619, $3 \mu \mathrm{M}$ TSA, $50 \mathrm{mM}$ NAM, and $2 \mathrm{mM}$ EDTA). Then, the same volume of Tris-saturated phenol (pH 8.0) was added into 5-mL centrifuge tube and mixed on a vortex for $5 \mathrm{~min}$. After centrifugation $\left(4^{\circ} \mathrm{C}, 10 \mathrm{~min}, 5,000 \mathrm{~g}\right)$, the upper phenol phase was transferred to a new centrifuge tube and added at least four volumes of ammonium sulfate-saturated methanol and incubated at $-20^{\circ} \mathrm{C}$ for at least $6 \mathrm{~h}$ to precipitate proteins. After centrifugation at $4^{\circ} \mathrm{C}$ for $10 \mathrm{~min}$, the supernatant was discarded. The remaining precipitate was washed with icecold methanol once, followed by ice-cold acetone for three times. The protein was redissolved in $8 \mathrm{M}$ urea and the protein concentration was determined with BCA kit according to the manufacturer's instructions.

\section{Protein tryptic digestion}

The disulfide bond of inner/inter-proteins were destroyed using $5 \mathrm{mM}$ dithiothreitol for $30 \mathrm{~min}$ at $56^{\circ} \mathrm{C}$ and alkylated with $11 \mathrm{mM}$ iodoacetamide for $15 \mathrm{~min}$ at room temperature in darkness. The protein sample was then diluted by adding $100 \mathrm{mM}$ TEAB to urea concentration less than 2M. Finally, trypsin was added at 1:50 trypsin-to-protein mass ratio for the first digestion overnight and 1:100 trypsin-to-protein mass ratio for a second digestion of $4 \mathrm{~h}$.

\section{HPLC fractionation and affinity enrichment}

HPLC separation process mainly refers to the research methods of Meng et al. (2019). The tryptic peptides were fractionated into fractions by high $\mathrm{pH}$ reverse-phase HPLC using Thermo Betasil $\mathrm{C}_{18}$ column $(5 \mu \mathrm{m}$ particles, $10 \mathrm{~mm}$ ID, $250 \mathrm{~mm}$ length). Briefly, peptides were first divided into 60 fractions using the gradients of $8 \%-32 \%$ acetonitrile (pH 9.0) over $60 \mathrm{~min}$. Then, the peptides were merged into four fractions and dried by freeze-drying apparatus. Next, tryptic peptides dissolved in NETN buffer $(100 \mathrm{mM} \mathrm{NaCl}$, $1 \mathrm{mM}$ EDTA, 50 mM Tris- $\mathrm{HCl}, 0.5 \%$ NP-40, pH 8.0) were incu- 
bated with pre-washed antibody beads (source In Hangzhou Jingjie Biotechnology Co., Ltd., PTM Bio) at $4^{\circ} \mathrm{C}$ overnight with gentle shaking. Then the beads were washed four times with NETN buffer and twice with $\mathrm{H}_{2} \mathrm{O}$, respectively. The bound peptides were eluted from the beads with $0.1 \%$ trifluoroacetic acid. Finally, the eluted fractions were combined and vacuum-dried. For LC-MS/MS analysis, the resulting peptides were desalted with $\mathrm{C}_{18}$ ZipTips (Millipore) according to the manufacturer's instructions.

\section{LC-MS/MS analysis and database searching}

The tryptic peptides were dissolved in $0.1 \%$ formic acid (solvent A), loaded onto a home-made reversed-phase analytical column (15 cm length, $75 \mu \mathrm{m}$ i.d.). The gradient was comprised of an increase from $6 \%$ to $23 \%$ solvent B $(0.1 \%$ formic acid in $98 \%$ acetonitrile) over $26 \mathrm{~min}, 23 \%$ to $35 \%$ in $8 \mathrm{~min}$ and climbing to $80 \%$ in $3 \mathrm{~min}$, then holding at $80 \%$ for the last $3 \mathrm{~min}$, all at a constant flow rate of $400 \mathrm{~nL} \mathrm{~min}^{-1}$ on an EASY-nLC 1000 UPLC system. The electrospray voltage applied was $2.0 \mathrm{kV}$. The $\mathrm{m} / \mathrm{z}$ scan range was 350 to 1,800 for full scan, and intact peptides were detected in the Orbitrap at a resolution of 70,000. Peptides were then selected for MS/ MS using NCE setting as 28 and the fragments were detected in the Orbitrap at a resolution of 17,500.

The resulting MS/MS data were processed using Maxquant search engine (v. 1.6.6.0). Tandem mass spectra were searched against Eucommia ulmoides_BIGD (26,722 proteins) database concatenated with reverse decoy database. The mass tolerance for precursor ions was set as $40 \mathrm{ppm}$ in First search and $5 \mathrm{ppm}$ in Main search, and the mass tolerance for fragment ions was set as 0.04 Da. Cysteine alkylation was set as a fixed modification, a variable modification was oxidation of methionine, acetylation of the $\mathrm{N}$-terminus of the protein, deamidation (NQ), and succinylation of lysine. FDR was adjusted to $<1 \%$ and minimum score for modified peptides was set $>40$.

\section{Bioinformatics analysis}

1. Motif analysis. Motif-x algorithm (MoMo) was used to identify the conserved motif of 20 amino acid sequence contained the 10 upstream and 10 downstream amino acids of modification sites, and the minimum number of occurrences was set to 20 .

2. Protein annotation. Gene Ontology (GO) annotation proteome was derived from the UniProt-GOA database (www. http://www.ebi.ac.uk/GOA/). Firstly, the corresponding UniProt IDs of identified protein IDs were mapped to GO IDs. The InterProScan software was used to annotate some proteins that were not annotated by UniProt-GOA based on the blastp method.

3. KEGG pathway annotation. Kyoto Encyclopedia of Genes and Genomes (KEGG) database was used to annotate the metabolic pathways involved in identified proteins. Firstly, KAAS was used to annotate the description of identified proteins in KEGG database. Then, map the annotation result on the KEGG pathway database using KEGG online service tools KEGG mapper.

4. GO/KEGG pathway functional enrichment analysis. For each GO category, proteins were classified by GO annotation into three categories: biological process, cellular compartment and molecular function. For each category, a twotailed Fisher's exact test was employed to test the enrichment of the identified modified protein against all proteins of the species database. The GO with a corrected p-value $<0.05$ is considered significant.
KEGG database was used to identify enriched pathways by a two-tailed Fisher's exact test to test the enrichment of the identified modified protein against all proteins of the species database. The pathway with a corrected p-value $<0.05$ was considered significant. These pathways were classified into hierarchical categories according to the KEGG website.

5. Protein-protein interaction (PPI) network analysis. All differentially expressed modified protein database accessions or sequences were searched against the STRING database v. 10.5 for protein-protein interactions. Cytoscape software (v. 3.0.1) was used visualize interaction networks from STRING.

\section{Results and discussion}

\section{Proteome-wide analysis of lysine succinylation sites on the proteins of $E$. ulmoides}

E. ulmoides is a medical and valuable economic tree species producing considerable secondary metabolites and high-quality gum. Lysine succinylation, as a newly identified protein post-translational modification (PTM), plays an important role in cellular physiology in some plants. However, the study of lysine succinylation in E. ulmoides is unclear. To explore the molecular mechanism of lysine succinylation on the growth and development of E. ulmoides, we performed the analysis of whole-proteome lysine-succinylation using LC-MS/MS in combination with bioinformatics tools. In this study, a total of 54,011 secondary spectra were obtained by mass spectrometry (Figure 1a).

The secondary spectrum of the mass spectrum was used to search the potential best-hit protein in protein theoretical data. The 9,272 spectra (17.2\%) of 54,011 secondary spectra corresponding 6,189 peptides were identified. On the other hand, the succinylated peptide were identified approximately $10.55 \%$ of identified peptides $(653$ out of 6,189 ). We employed criterion of localization probability $>0.75$ to filter the identification data to guarantee the high reliability of the results. The identified 653 succinylation sites were derived from the 365 proteins. In addition, we further compared the amount and distribution of succinylated protein, found that the number of succinylated protein in E. ulmoides was larger than that in other plants, such as patchouli plant, D. officinale, strawberry, etc. But it was much lower than other non-plant organisms (E. coli, Mycobacterium tuberculosis, H. sapiens (HeLa), M. musculus (liver)) (Wang et al., 2019; Feng et al., 2017; Fang et al., 2018; Xu et al., 2017; Zhang et al., 2010; Xie et al., 2015; Du et al., 2011). First, the reasons for differences in the number of succinylation sites and succinylation proteins reported plant species may be related to the species specificity, and the growth status, growth environment, and sampling location of the test subject may also relate to differences. Second, the reason for the relatively low number of succinylation sites and succinylation proteins may be related to the evolution of organisms, and more research is needed to explore the reasons.

To confirm the accuracy of the MS data, we verified the mass errors of all identified peptides in MS data. We found that first-order mass error of most spectra of identified proteins is less than $10 \mathrm{ppm}$ consisting with the high-accuracy characteristics of the orbital trap mass spectrometer (Figure 1b). The numbers of modification sites in each protein was further calculated to explore the enrichment of succinylation sites in the identified proteins from E. ulmoides. The number of succinylated sites in each protein varied from 1 to 11 , which is positive related to the protein length. Out of the 


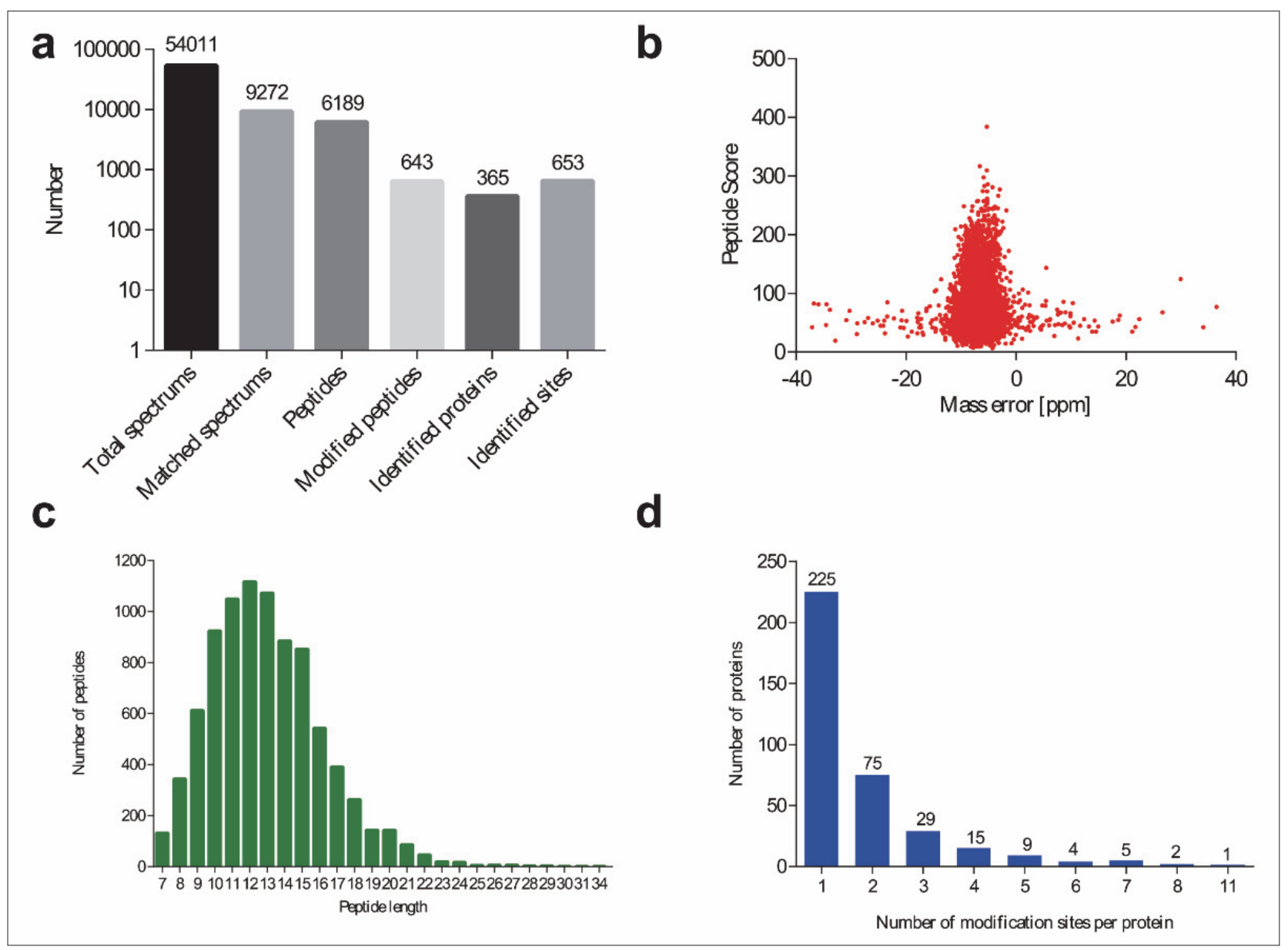

FIGURE 1. Profile of identified succinylated sites, peptides and proteins. (a) Basic statistical chart of mass spectrometry data results. (b) The distribution of mass error. (c) Length distribution of the identified peptides. (d) Distribution of succinylated sites in succinylated proteins.

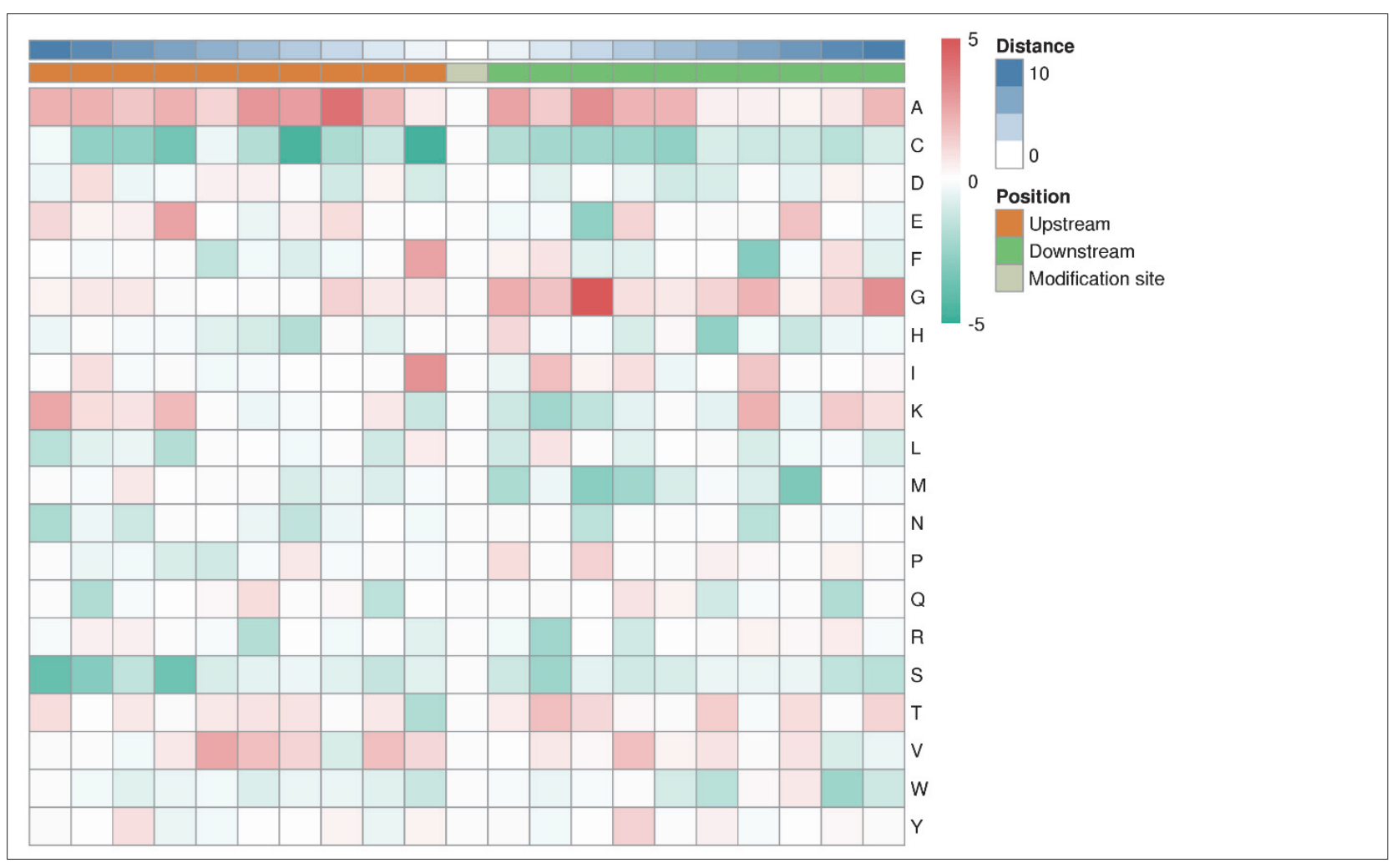

FIGURE 2. Heat map of the amino acids flanking the succinylation sites from 10 amino acids downstream to 10 amino acids upstream of succinylated lysine site. 
365 succinylated proteins, approximately $61.6 \%(225 / 365)$ modified proteins had only one succinylated site, and $20.5 \%$ (75/365) contained two succinylated sites, $17.8 \%(65 / 365)$ had more than two succinylated sites, and the average succinylation modification level was 1.79 (653/365) (Figure 1c). Remarkably, oxygen-evolving enhancer protein 1 , chloroplastic-like contained the most lysine succinylation modification site (11).

More modification sites may have special regulatory effects on the function of the protein, which requires further experiments to verify. In this study, all succinylated modified peptides were composed of 7-29 amino acids, of which 7-20 amino acids were mainly concentrated, which conformed to the rule of trypsin enzymatic hydrolysis and HCD fragmentation (Figure 1d). The results showed that the distribution of peptide lengths met quality control requirements in this experiment.

\section{Motif analysis of lysine succinylation sites}

To investigate the features of lysine succinylation sites of $E$. ulmoides, we analyzed amino acid sequences from -10 to +10 of the 653 succinylation sites using MoMo software. In this study, a total of four consensus sequence motifs were identified and listed, AXXKsuc, KsucXXG, IKsuc and FKsuc (Ksuc indicates a succinylated lysine, while $\mathrm{X}$ indicates an unspecified amino acid residue). The heat map was used to show the motif enrichment of the upstream and downstream amino acids of the lysine succinylation site. Our results showed that the frequencies of alanine (A) in position -3, gly- cine $(\mathrm{G})$ in position +3 , isoleucine (1) and phenylalanine $(\mathrm{F})$ in position -1 (Figure 2).

Compared with the conserved motifs in other plant species, the motifs IKsuc and FKsuc were also significantly enriched in the succinylated peptides in strawberry, and AXXKsuc was also observed in tomato (Fang et al., 2018; Jin et al., 2016). These three motifs were also distributed in other species, indicating that these motifs conservatively distributed near succinylated lysine sites during the evolution of land plants. A new specific motif (KsucXXG) was only identified in E. ulmoides based on the analysis of lysine succinylation of land plants. Furthermore, the glycine $(G)$ was commonly enriched near the succinylation site among the species that had conducted the succinylome analysis, such as patchouli plants, silkworm and Candida albicans (Wang et al., 2019; Chen et al., 2018; Zheng et al., 2016). This may be due to the small steric hindrance caused by amino acids with smaller side chain groups, which is more conducive to succinyl groups with the large molecular weight attached to the side chain of lysine residues.

\section{Function classification and subcellular location analysis}

To better elucidate the potential function of lysine-succinylated proteins in E. ulmoides, the GO database which is commonly used for gene/gene-product annotation was used to annotate the identified proteins with at least one succinylated site. All succinylated proteins were unevenly distributed in three GO categories (Biological process, Cellular component, and Molecular function). In the Biological process,

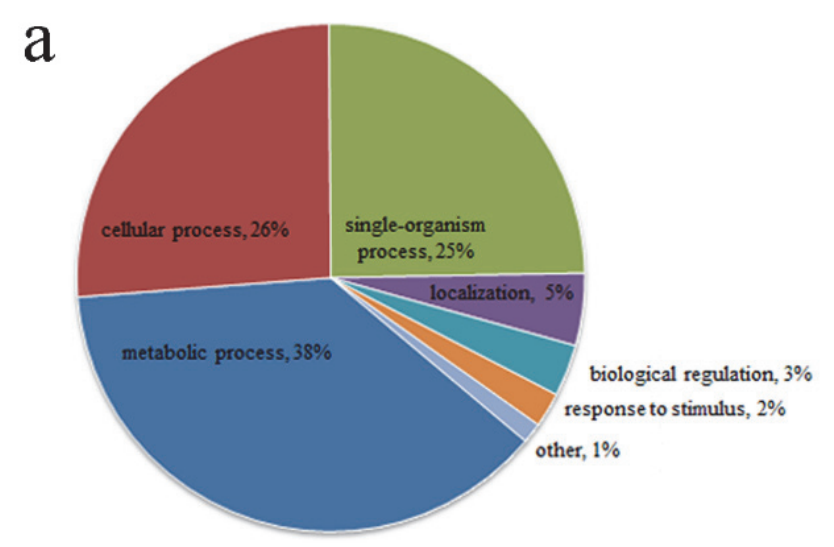

b

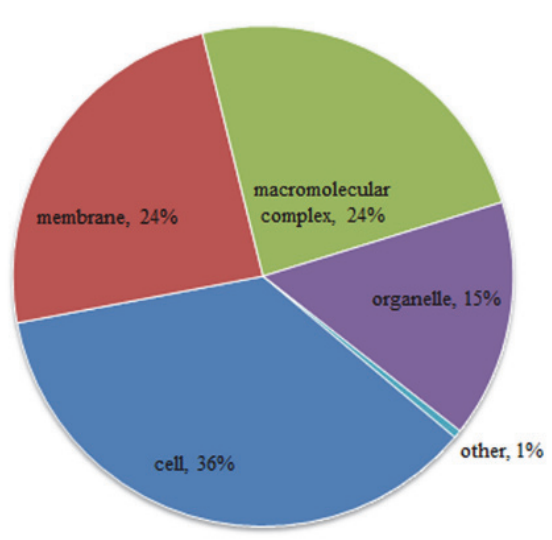

C

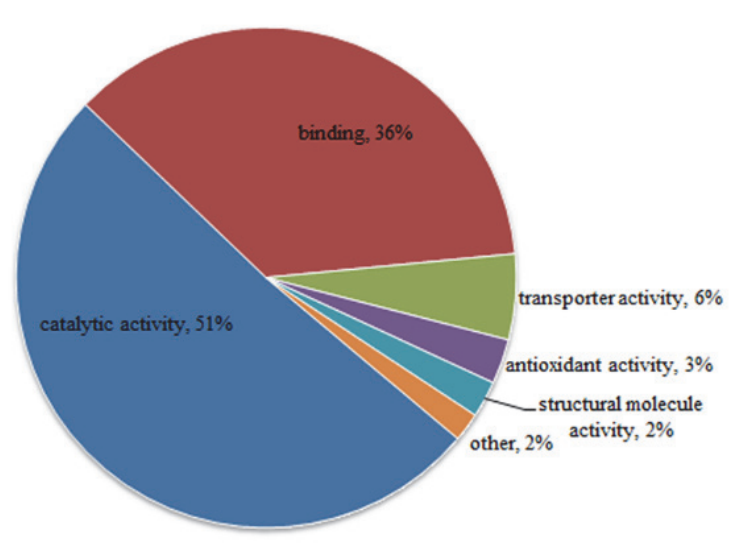

d

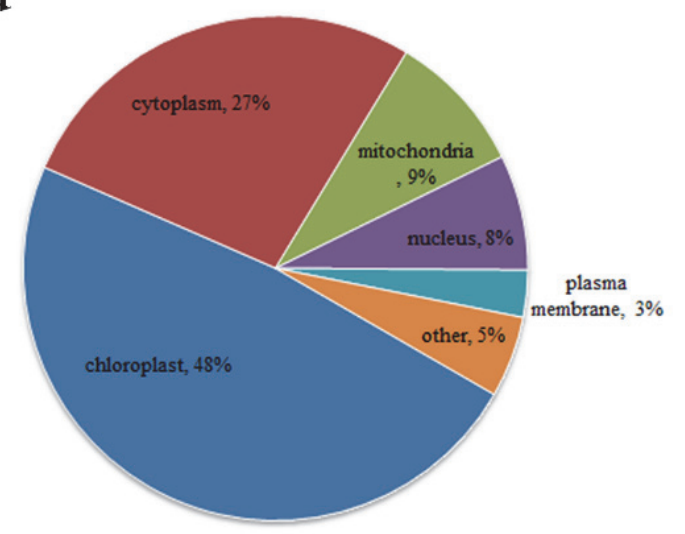

FIGURE 3. Profile of identified succinylated sites, peptides and proteins. (a) Classification of the succinylated proteins based on biological process. (b) Classification of the succinylated proteins based on molecular function. (c) Classification of the succinylated proteins based on molecular function. (d) Subcellular localization of the succinylated proteins. 
the significantly enriched GO terms were metabolic process (38\%), cellular process (26\%) and single-organism process (25\%), while localization, biological regulation, response to stimulus, and the other terms accounted for 5, 3, 2 and 1\%, respectively (Figure 3a). In cellular component, the modified proteins were distributed in the cell (36\%), membrane (24\%), macromolecular complex (24\%), organelle (15\%) and other (1\%), respectively (Figure 3b). Consistently, in the GO term of molecular function, proteins related to catalytic and binding activities were the most prevalent modified proteins in E. ulmoides, accounting for 51 and $36 \%$ of all the succinylated modified protein, respectively (Figure 3c).

Protein subcellular localization prediction is an important component of bioinformatics. Identifying the subcellular locations of proteins can improve our understanding of their functions and mechanisms of molecular interaction. Thus, the subcellular localization of the succinylated proteins in E. ulmoides were predicted, found that $48 \%$ of the succinylated proteins (176/365) were located in the chloroplast, and $27 \%$ of the succinylated proteins were located in the cytosol and $9 \%$ of the succinylated proteins were mitochondrialocated (Figure 3d). The above results indicated that lysine succinylated proteins were widely distributed in some organelle that were involved in energy metabolism in E. ulmoides.

Compared to other plants, the GO annotation and subcellular localization of succinylated proteins in E. ulmoides showed similar distribution, and only differences existed in detailed values of each item. However, in mammals and bacteria, most succinylated modified proteins are mainly concentrated in mitochondria, cytoplasm and nucleus (Xie et al., 2012; Zhang et al., 2010). For example, in Toxoplasma gondii, the mitochondria contained the most succinylated modified protein $(26 \%)$, while in E. ulmoides only $9 \%$ of succinylated modified protein was located in the mitochondria (Li et al.,
2014). This may be because plants as producers use photosynthesis to convert solar energy into the chemical energy of organic matter, and provide energy (organic matter) and oxygen for other organisms. They play an important role in the ecosystem, and the chloroplast is the main place for photosynthesis. Therefore, the proportion of succinylated proteins in chloroplasts is relatively high, and it also further illustrates that succinylation may play a special role in plant photosynthesis.

\section{Functional enrichment analyses of lysine succinylated proteins}

In order to investigate the significant enrichment trend of succinylated proteins in E. ulmoides, we conducted enrichment analysis at three levels: GO classification, KEGG pathway, and protein domain. GO enrichment analysis showed that most of the succinylated proteins were mainly related to metabolic processes, energy generation and biosynthesis processes of biological process (Figure 4a). In addition, Ribose phosphate metabolic process, nucleoside metabolic process, oxoacid metabolic process, and carboxylic acid metabolic process were the top four significantly enriched molecular functions, implying that the metabolism of many important substances in cells may be influenced by lysine succinylation. Meanwhile, significant enrichments of succinylated proteins involved in photosystem, thylakoid, thylakoid part and photosynthetic membrane were observed in the cellular component. These four GO terms were all related to chloroplast, suggesting that photosynthesis-related enzymes may be the targets and substrates of lysine succinylation modifications. Regarding the molecular function category, catalytic activity, oxidoreductase activity, cotactor binding and coenzyme binding were the top four enriched terms.

KEGG analysis was conducted to investigate the succi-

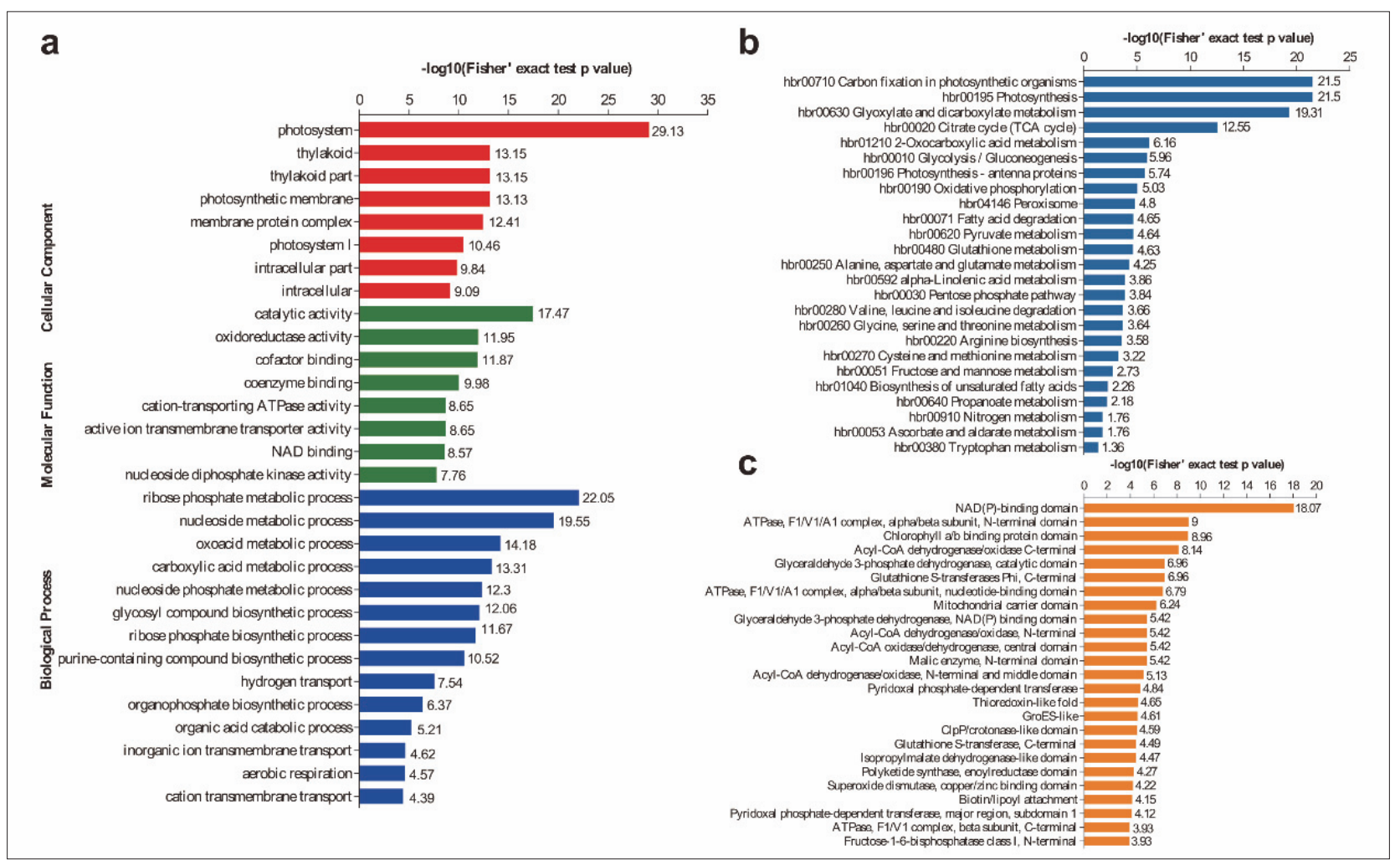

FIGURE 4. Enrichment analysis of succinylated proteins based on their annotation. (a) GO enrichment analysis of succinylated proteins in Eucommia ulmoides. (b) KEGG enrichment analysis of succinylated proteins in E. ulmoides. (c) Domain enrichment analysis of succinylated proteins in E. ulmoides. 
nylated proteins involved in metabolic pathways in E. ulmoides. A total of 25 significantly enriched pathways (Figure $4 \mathrm{~b}$ ) were identified. Combined with the GO enrichment analysis (cellular component), KEGG analysis showed that carbon fixation in photosynthetic organisms and photosynthesis were top two of the most significantly enriched pathway, suggesting the regulatory role of succinylation in photosynthesis. In addition, Glyoxylate and dicarboxylate metabolism and Citrate cycle (TCA cycle) related to energy metabolism were highly enriched.

The results of domain enrichment analysis showed that proteins containing domains of NAD(P)-binding domain were the most enrichment in succinylated proteins (Figure 4c). Moreover, proteins contained one or more domains of ATPase, F1/V1/A1 complex, alpha/beta subunit, N-terminal domain and chlorophyll $a / b$ binding protein were also observed. Previous studies had revealed that these proteins contained above domains were involved in energy metabolism, which was consistent with the GO and KEGG enrichment analysis. The above research shows that lysine succinate protein is widely distributed in multiple pathways, indicating that this post-translational modification plays an important role in cell metabolism.

\section{Protein-protein interaction (PPI) network construction of succinylated proteins}

PPIs are widespread in all cell types, which are essential for different biological processes (Wang et al., 2013). To fur- ther screen out node succinylated proteins, we constructed a PPI network for 365 succinylated proteins through the STRING database using the Cytoscape software. After comparing with the STRING (v. 10.5) protein network interaction database, 191 succinylated proteins were selected as nodes to form the PPI network of E. ulmoides. Several highly enriched interactive clusters and perfect interactive networks were extracted from 191 succinylated proteins by the MCODE plug-in toolkit, which are related to the citrate cycle (TCA cycle), photosynthesis, carbon fixation, and glycolysis (Figure 5). Construction and analysis of the succinylated proteins interaction network indicates that lysine succinylation of proteins plays an important role in the energy conversion process in plants.

\section{Analysis of succinylated proteins involved in energy metabolism}

Based on the results of PPIs, subcellular localization and functional enrichment analysis, we found that many succinylated proteins were related to metabolism processes in E. ulmoides, such as TCA cycle, photosynthesis, carbon fixation and glycolysis. In order to further explore the role of succinylation in these processes, the succinylated proteins contained in these pathways were analyzed.

The photosynthesis that occurs in chloroplasts utilizes light energy to convert carbon dioxide and water into carbohydrates with energy storage to maintain plant growth and development (Zhang et al., 2016). Our results showed that

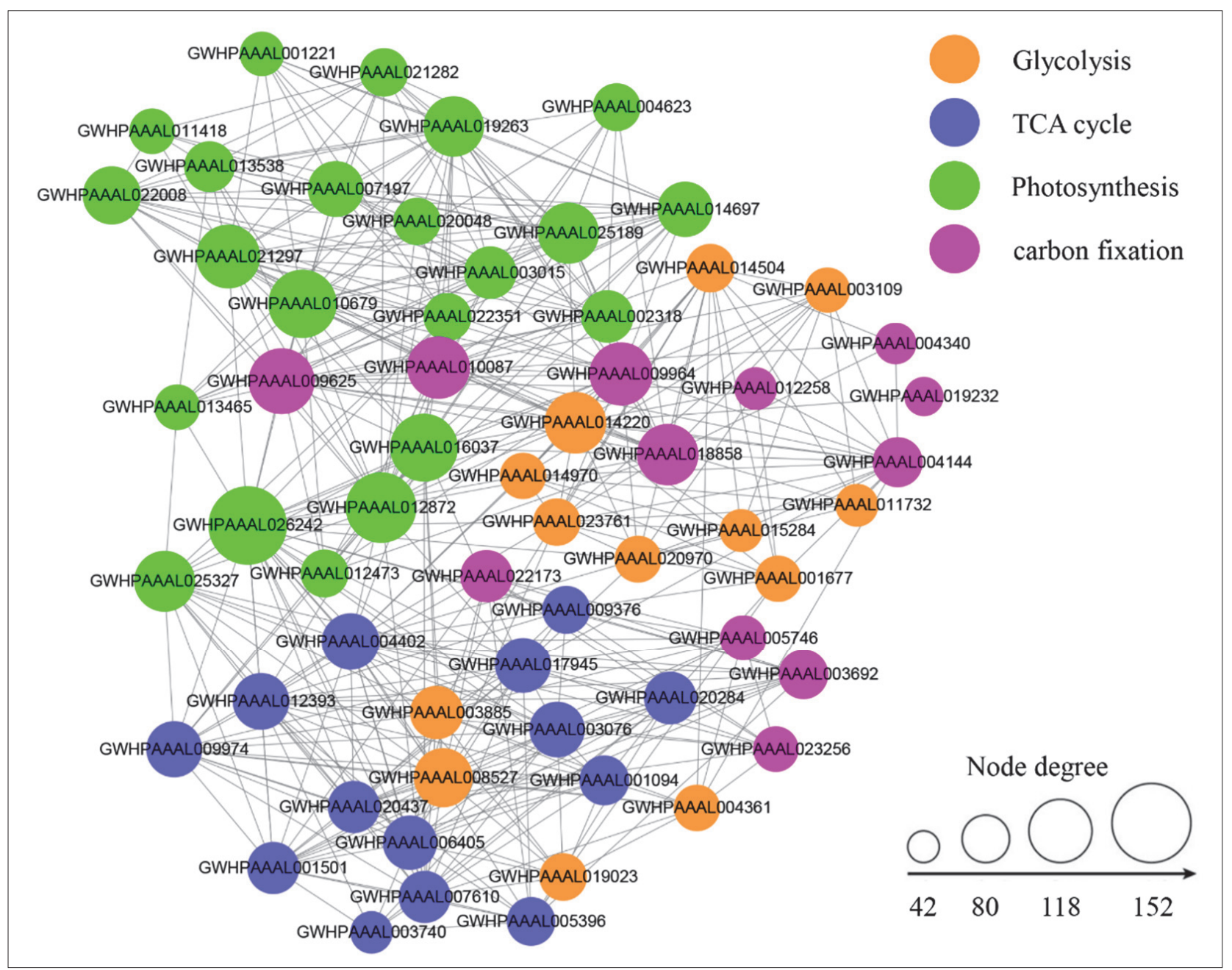

FIGURE 5. Interaction networks of succinylated proteins in Eucommia ulmoides. 
$48.22 \%$ of the succinylated proteins in E. ulmoides are located to the chloroplast (Figure 3d). This phenomenon is consistent with the above enrichment of succinylated proteins in energy metabolic processes (Figure 3), suggesting that lysine succinylation may play an important role in photosynthesis.
This result was consistent with previous studies that lysine succinylated proteins mapped to the photosynthesis pathway, indicating a potentially conserved function for lysine succinylation in the regulation of photosynthesis among different plant species (He et al., 2016; Shen et al., 2016).

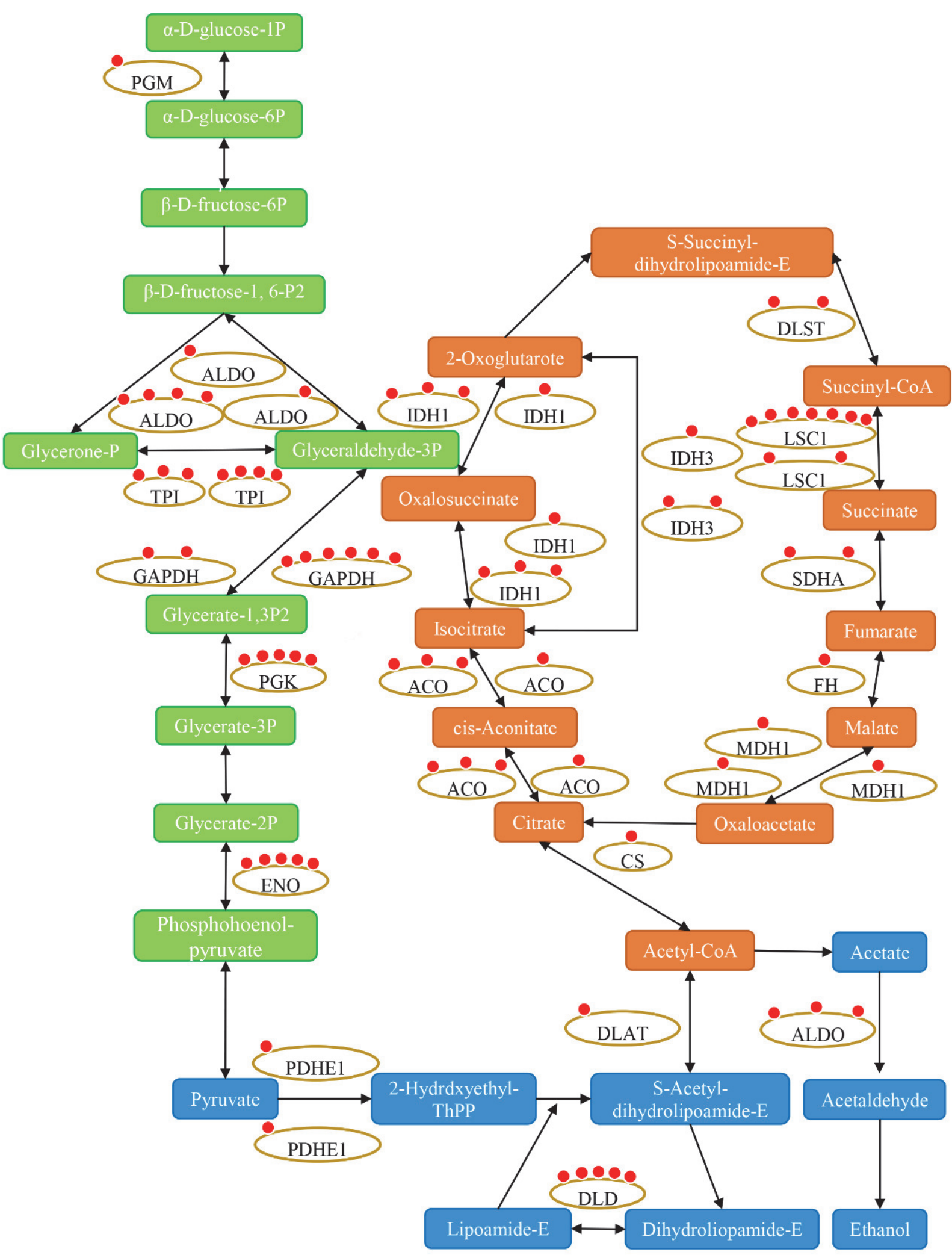

FIGURE 6. Succinylated enzymes involved in major metabolic pathways (glycolysis, pyruvate metabolism and the TCA cycle). 
Furthermore, we performed the blast to annotate the identified succinylated proteins, twenty-three succinylated modified proteins were identified to be involved in photosynthesis in E. ulmoides, including nine subunits of Photosystem I (PsaA, PsaB, PsaD, PsaE, PsaF, PsaG, PsaH, PsaL, PsaN), seven subunits of Photosystem II (PsbB, PsbO, PsbP, PsbQ PsbR, PsbS, Psb27), three subunits of ATP synthase (beta, alpha, gamma) and only one subunit of cytochrome b6f complex (PetC). In addition, lysine succinylated FNR (FerredoxinNADP + oxidoreductase) catalyzing the reversible electron transfer between Fd and NAD(P)H was identified, which was also distributed in wheat (Mulo, 2011; Zhang et al., 2017). Oxygen-evolving complex (OEC) is a complex of chloroplast photosystem II, located inside the cavity of the thylakoid membrane, which is indispensable for oxidizing water molecules to release oxygen during chloroplast photoreaction. OEC is composed of three polypeptides with apparent molecular weights of $33 \mathrm{kDa}, 23 \mathrm{kDa}$, and $17 \mathrm{kDa}$ (corresponding to proteins: psbO, psbP, and psbQ) and some peptides with smaller molecular weights that have not been determined. Among them, the psbO (33 kDa) protein has a protective effect on the manganese clusters, which are core components involved in photosynthetic oxygen release, and is called a manganese stabilizing protein (MSP). The psbP (23 kDa) and psbQ (17 kDa) proteins bind to $\mathrm{Ca}^{2+}$ and $\mathrm{Cl}^{-}$during the process of photo-oxidizing water to release oxygen, respectively (Seidler, 1996; Yu et al., 2001). In this study, three proteins (psb0, psbP, and psbQ) generated an OEC complex, which was modified by succinylation with more modification sites. For example, the psbO and psbQ proteins have 11 and 8 modification sites, respectively, suggesting that more succinylation modification sites may be of great significance for better maintaining the function of OEC.
Carbon fixation is an important process in plant metabolism, which utilizes products of photosynthesis, such as ATP and NADPH to fix carbon for manufacture of energy materials to maintain plant life activities (Spreitzer et al., 2002; Lara et al., 2003; Minagawa, 2011). In the carbon fixation pathway, 62 lysine succinylation modification sites were identified and mapped to 23 proteins (Table 1). Interestingly, the ribulose-bisphosphate carboxylase large chain (RbcL) contained only one succinylation modification site in this study, which was different from many previous studies that more succinylation modified sites were presented in ribulose bisphosphate carboxylase (Rubisco) catalyzing the limiting step of photosynthetic capacity (Farquhar et al., 1980; Zhou et al., 2017; Wang et al., 2019; Xu et al., 2017; Zhen et al., 2016), indicating succinylation modifications between different species may have their own specificity. Collectively, these findings indicate that lysine succinylation may play an indispensable role in regulating photosynthesis and carbon metabolism in E. ulmoides (Figure 6).

Glycolytic enzymes are recognised as key enzyme converting glucose into pyruvate followed by acetyl-CoA which enters the tricarboxylic acid cycle. In this process, while providing energy for the body, many intermediate products are also produced for other metabolic activities (Fernie et al., 2004). Among them, succinic acid and succinyl-CoA serve as donors of succinyl which is an intermediate metabolite of the tricarboxylic acid cycle.

Increasing evidence shows that many succinylated proteins are involved in multiple metabolic pathways including glycolysis pathway, pyruvate metabolism and TCA cycle. In our study, six out of 11 glycolytic enzymes were identified as succinylated proteins (Table 2). Most of proteins are also succinylated in E. coli, Mycobacterium tuberculosis, mammals

TABLE 1. The lysine succinylation proteins in carbon fixation pathway.

\begin{tabular}{|c|c|c|c|}
\hline Accession number & Description & Abbreviation & Position \\
\hline GWHPAAAL022173 & Glutamate - glyoxylate aminotransferase & GGAT & $\begin{array}{l}\text { K39, K90, K463, K114, K122, K4, K357, } \\
\text { K16 }\end{array}$ \\
\hline GWHPAAAL003692 & Aspartate aminotransferase & GOT1 & K115, K297 \\
\hline GWHPAAAL009376 & Malate dehydrogenase 1 & $\mathrm{MDH} 1$ & K312 \\
\hline GWHPAAAL004402 & Malate dehydrogenase 2 & $\mathrm{MDH} 2$ & K236 \\
\hline GWHPAAAL017945 & Malate dehydrogenase 2 & $\mathrm{MDH} 2$ & K341 \\
\hline GWHPAAAL005746 & Malate dehydrogenase 1 & $\mathrm{MDH} 1$ & $\mathrm{~K} 227, \mathrm{~K} 80$ \\
\hline GWHPAAAL023256 & Malate dehydrogenase 1 & MDH1 & K294, K94 \\
\hline GWHPAAAL019232 & Ribulose-bisphosphate carboxylase large chain & $\mathrm{RbcL}$ & K21 \\
\hline GWHPAAAL014220 & Phosphoglycerate kinase & PGK & $\mathrm{K} 123, \mathrm{~K} 89, \mathrm{~K} 414, \mathrm{~K} 78, \mathrm{~K} 202$ \\
\hline GWHPAAAL011732 & Glyceraldehyde 3-phosphate dehydrogenase & GAPDH & K70, K310, K118, K264, K224, K220 \\
\hline GWHPAAAL015284 & Glyceraldehyde 3-phosphate dehydrogenase & GAPDH & $\mathrm{K} 77, \mathrm{~K} 70$ \\
\hline GWHPAAAL009964 & Glyceraldehyde-3-phosphate dehydrogenase (NADP+) & GAPA & $\mathrm{K} 308, \mathrm{~K} 281, \mathrm{~K} 283, \mathrm{~K} 80$ \\
\hline GWHPAAAL014504 & Fructose-bisphosphate aldolase, class I & ALDO & K17 \\
\hline GWHPAAAL012447 & Fructose-bisphosphate aldolase, class I & ALDO & K345, K375, K366, K79 \\
\hline GWHPAAAL003109 & Fructose-bisphosphate aldolase, class I & ALDO & K306 \\
\hline GWHPAAAL004340 & Fructose-1,6-bisphosphatase I & FBP & $\mathrm{K} 25, \mathrm{~K} 75$ \\
\hline GWHPAAAL018858 & Fructose-1,6-bisphosphatase I & FBP & K407 \\
\hline GWHPAAAL004144 & Transketolase & - & K479, K605, K132, K594, K587 \\
\hline GWHPAAAL010087 & Sedoheptulose-1,7-bisphosphatase & SBPase & K336, K258 \\
\hline GWHPAAAL012258 & Ribose 5-phosphate isomerase $\mathrm{A}$ & RPIA & K137, K37 \\
\hline GWHPAAAL009625 & Phosphoribulokinase & PRK & $\mathrm{K} 230, \mathrm{~K} 223$ \\
\hline GWHPAAAL020970 & Triosephosphate isomerase & TPI & K197, K4, K120 \\
\hline GWHPAAAL014970 & Triosephosphate isomerase & TPI & K181, K256, K321, K263 \\
\hline
\end{tabular}


TABLE 2. The lysine succinylation proteins in the glycolysis pathway, pyruvate metabolism and TCA cycle.

\begin{tabular}{|c|c|c|c|}
\hline Accession number & Description & Abbreviation & Position \\
\hline GWHPAAAL001677 & Phosphoglucomutase & PGM & K545 \\
\hline GWHPAAAL014504 & Fructose-bisphosphate aldolase & ALDO & K17 \\
\hline GWHPAAAL012447 & Fructose-bisphosphate aldolase & ALDO & K345, K375, K366, K79 \\
\hline GWHPAAAL003109 & Fructose-bisphosphate aldolase & ALDO & K306 \\
\hline GWHPAAAL020970 & Triosephosphate isomerase & TPI & K197, K4, K120 \\
\hline GWHPAAAL014970 & Triosephosphate isomerase & TPI & K70, K310, K118, K264, K224, K220 \\
\hline GWHPAAAL011732 & Glyceraldehyde 3-phosphate dehydrogenase & GAPDH & $\mathrm{K} 70, \mathrm{~K} 310, \mathrm{~K} 118, \mathrm{~K} 264, \mathrm{~K} 224, \mathrm{~K} 220$ \\
\hline GWHPAAAL015284 & Glyceraldehyde 3-phosphate dehydrogenase & GAPDH & $\mathrm{K} 77, \mathrm{~K} 70$ \\
\hline GWHPAAAL014220 & Phosphoglycerate kinase & PGK & K123, K89, K414, K78, K202 \\
\hline GWHPAAAL023761 & Enolase & ENO & K137 \\
\hline GWHPAAAL003885 & Pyruvate dehydrogenase E1 component & PDHE1 & K289 \\
\hline GWHPAAAL004361 & Pyruvate dehydrogenase E1 component & PDHE1 & K344 \\
\hline GWHPAAAL019023 & Pyruvate dehydrogenase E2 component & DLAT & K380 \\
\hline GWHPAAAL008527 & Dihydrolipoamide dehydrogenase & DLD & K215, K503, K178, K199, K277 \\
\hline GWHPAAAL009065 & Aldehyde dehydrogenase & ALDH & K104, K453, K518 \\
\hline GWHPAAAL003076 & Citrate synthase & CS & K77 \\
\hline GWHPAAAL003740 & Aconitate hydratase & ACO & K522, K256, K187 \\
\hline GWHPAAAL002002 & Aconitate hydratase & ACO & K250 \\
\hline GWHPAAAL020284 & Isocitrate dehydrogenase & IDH1 & K456, K296, K446 \\
\hline GWHPAAAL005396 & Isocitrate dehydrogenase & IDH1 & K240 \\
\hline GWHPAAAL001094 & 2-oxoglutarate dehydrogenase E2 component & DLST & K307, K220 \\
\hline GWHPAAAL007610 & Succinyl-CoA synthetase alpha subunit & LSC1 & K119, K59, K205, K197, K188, K262, K100 \\
\hline GWHPAAAL009974 & Succinyl-CoA synthetase alpha subunit & LSC1 & K66, K305 \\
\hline GWHPAAAL012393 & Succinate dehydrogenase & SDHA & K169, K323 \\
\hline GWHPAAAL020437 & Fumarate hydratase & $\mathrm{FH}$ & K242 \\
\hline GWHPAAAL009376 & Malate dehydrogenase & MDH1 & K312 \\
\hline GWHPAAAL004402 & Malate dehydrogenase & MDH1 & K236 \\
\hline GWHPAAAL017945 & Malate dehydrogenase & MDH1 & K341 \\
\hline GWHPAAAL006405 & Isocitrate dehydrogenase (NAD+) & IDH3 & K191, K172 \\
\hline GWHPAAAL001501 & Isocitrate dehydrogenase (NAD+) & IDH3 & K110 \\
\hline
\end{tabular}

and plants such as Taxus $\times$ media, rice and Dendrobium officinale, indicating the potential regulatory role of lysine succinylation in glycolytic metabolism (Zhang et al., 2010; Xie et al., 2015; Du et al., 2011; Shen et al., 2016; He et al., 2016; Feng et al., 2017). Furthermore, conversion of pyruvate to acetyl-CoA for TCA needed to be catalyzed by the pyruvate dehydrogenase complex. In this complex, five components were found to be succinylated at lysine (Figure 5). Previous studies in bacteria (E. coli), yeast (S. cerevisiae), human cells and mouse liver tissue found lysine succinylation on TCA cycle-related enzymes and revealed potential conserved function of succinylation in the regulation of TCA cycle (Weinert et al., 2013). Consistent with this previous study, ten key enzymes were lysine-succinylated suggesting that lysine succinylation may participate in TCA cycle regulation in this herb (Figure 6). A study in yeast showed that succinylation levels could be elevated by up-regulating $\alpha$-ketoglutarate dehydrogenase catalyzing the formation of succinyl-CoA or downregulating succinyl-CoA ligase catabolizing the succinyl-CoA in TCA cycle, further indicating that the production of succinyl-CoA in TCA cycle may be associated with succinylation levels throughout the cells (Meng et al., 2019). In this study, these two enzymes were all modified by succinylation, in which Lsc1 has 7 modification sites, which indicates that it may have a regulating effect on the overall succinylation level of E. ulmoides.

\section{Conclusions}

In this study, a total of 653 lysine succinylation sites distributed in 365 proteins were identified and analysed. The succinylated proteins were widely distributed in the physiological processes of $E$. ulmoides, and especially in metabolic processes associated with energy. The subcellular localization and pathway enrichment analysis results showed that $84 \%$ of the modified proteins were concentrated in chloroplast, cytoplasm and mitochondria, which were involved in the photosynthesis, carbon fixation, glycolysis and TCA cycle of E. ulmoides. The above pathways together constitute the two-part process of energy supply for plant survival, namely conversion of solar energy into biomass energy for storage in plants and conversion of energy-stored substances in plants into energy (ATP) for direct use by plants. Combined with the reported studies on succinylation of other plants, the results are similar to those in this study, like patchouli plants. In summary, it can be further speculated that lysine succinate modification may play an important role in providing energy for the growth and development of plants. The dataset may serve as a rich resource that can be used to examine the functions of lysine succinylation in this important medicinal herb. 


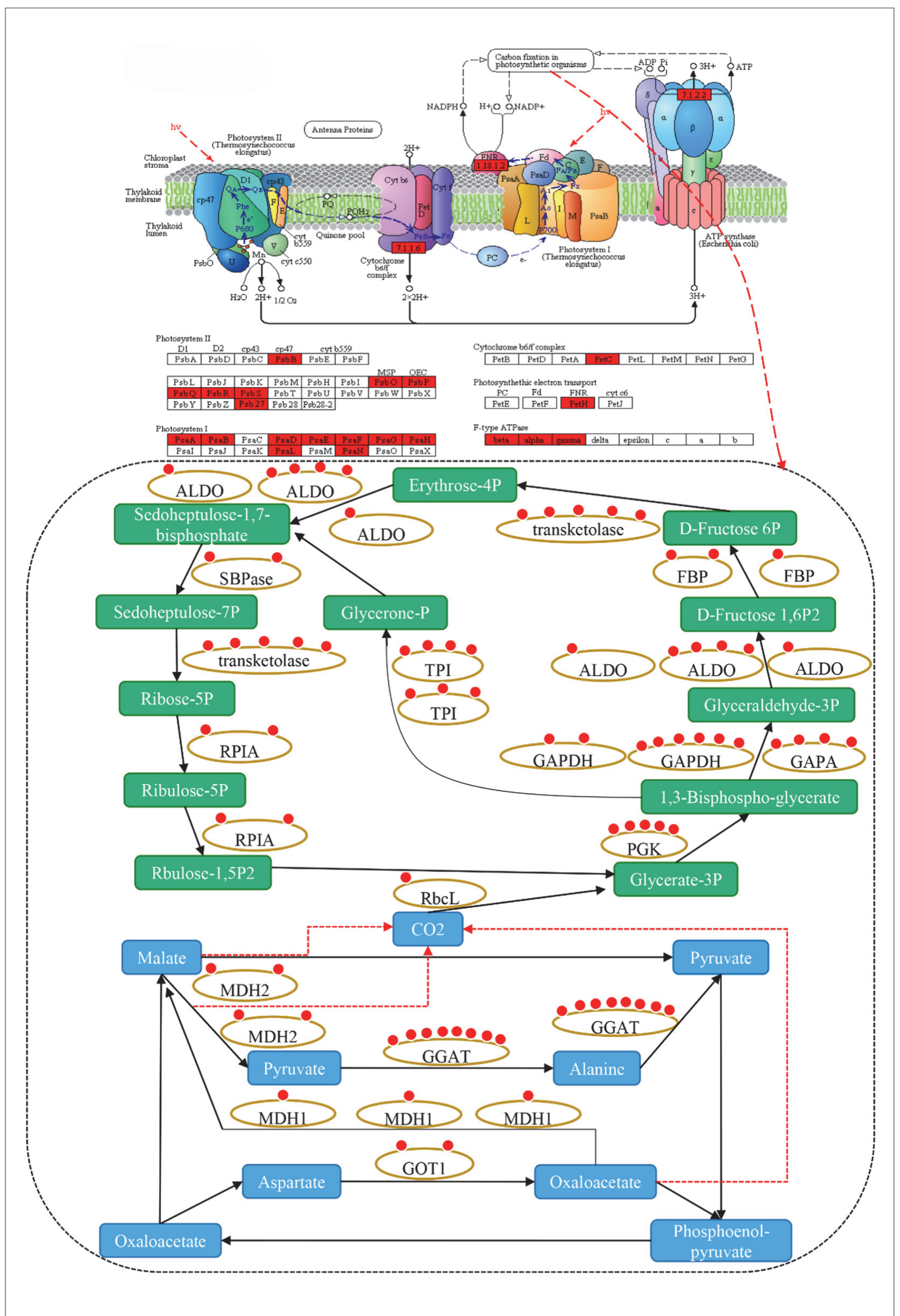

FIGURE 7. Succinylated enzymes involved in major metabolic pathways (carbon fixation) and photosynthesis. 


\section{Funding}

This work was financially supported by Guizhou Tea Industry Technology Innovation Center (Qianke Zhongyindi [2017] 4005) and by Excellent Young Scientific and Technological Talent Program of Guizhou Province "Research on Key Techniques for Improving the Quality of Albino, Yellowing and Purpleing Tea Tree Varieties" (Qianke Hepingtairencai [2019] 5651).

\section{References}

Bamba, T., Fukusaki, E.I., and Kobayashi, N.A. (2002). In-situ chemical analyses of trans-polyisoprene by histochemical staining and Fourier transform infrared microspectroscopy in a rubber-producing plant, Eucommia ulmoides Oliver. Planta 21, 934-939. https://doi.org/ 10.1007/s00425-002-0832-3.

Cantin, G.T., and Yates III, J.R. (2004). Strategies for shotgun identification of post-translational modifications by mass spectrometry. J. Chromatography A. 1053, 7-14. https://doi.org/ 10.1016/j.chroma.2004.06.046.

Chen, J., Li, F.B., Liu, Y., Shen, W.F., Du, X., He, L.H., Meng, Z.Q., Ma, X.L., and Wang, Y.Q. (2018). Systematic identification of mitochondrial lysine succinylome in silkworm (Bombyx mori) midgut during the larval gluttonous stage. J. Proteomics 174, 61-70. https://doi.org/ 10.1016/j.jprot.2017.12.019.

Chen, R., Namimatsu, S., Nakadozono, Y., Bamba, T., Nakazawa, Y., and Gyokusen, K. (2008). Efficient regeneration of Eucommia ulmoides from hypocotyl explant. Biolog. Plant. (Prague) 52, 713-717. https:// doi.org/ 10.1023/A:1025604713256.

Cheng, Y., Hou, T.L., Ping, J., Chen, G.F., and Chen, J.J. (2016). Quantitative succinylome analysis in the liver of non-alcoholic fatty liver disease rat model. Proteome Sci. 14, 3. https://doi.org/ 10.1186/s12953-016-0092-y.

Choudhary, C., Kumar, C., Gnad, F., Nielsen, M.L., Rehman, M., Walther, T.C., Olsen, J.V., and Mann, M. (2009). Lysine acetylation targets protein complexes and co-regulates major cellular functions. Science 325, 834-840. https://doi.org/10.1126/science.1175371.

Colak, G., Xie, Z.Y., Zhu, A.Y., Dai, L.Z., Lu, Z.K., Zhang, Y., Wan, X.L., Chen, Y., Cha, Y.H., Lin, H.N., et al. (2013). Identification of lysine succinylation substrates and the succinylation regulatory enzyme CobB in Escherichia coli. Molec. Cell. Proteomics 12, 3509-3520. https://doi.org/ 10.1074/mcp.M113.031567.

Dai, X.P., Huang, Q., Zhou, B.T., Gong, Z.C., Liu, Z.Q., and Shi, S.Y. (2013). Preparative isolation and purification of seven main antioxidants from Eucommia ulmoides Oliv. (Du-Zhong) leaves using HSCCC guided by DPPH-HPLC experiment. Food Chem. 139, 563-570. https://doi.org/ 10.1016/j.foodchem.2013.02.006.

Du, J.T., Zhou, Y.Y., Su, X.Y., Yu, J.J., Khan, S., Jiang, H., Kim, J., Woo, J., Kim, J.H., Choi, B.H., He, B., Chen, W., Zhang, S., Cerione, R.A., Auwerx, J., Hao, Q., and Lin, H. (2011). Sirt5 is a NAD-dependent protein lysine demalonylase and desuccinylase. Science 334, 806-809. https://doi. org/10.1126/science.1207861

Fang, X.P., Xin, Y., Sheng, Z.L., Liu, H., Jiang, A.L., Wang, F., Yang, J., Xi, X.J., Zha, Q., Zhang, L.Q., Dai, L.Y., Yan, C.Q., and Chen, J.P. (2018). Systematic identification and analysis of lysine succinylation in strawberry stigmata. J. Agric. Food Chem. 66, 13310-13320. https:// doi.org/ 10.1021/acs.jafc.8b02708.

Farquhar, G.D., Caemmerer, S.V., and Berry, J.A. (1980). A biochemical model of photosynthetic $\mathrm{CO}_{2}$ assimilation in leaves of $\mathrm{C}_{3}$ species. Planta 149, 78-90. https://doi.org/10.1007/BF00386231.

Feng, S.G., Jiao, K.L., Guo, H., Jiang, M.Y., Hao, J., Wang, H.Z., and Shen, C.J. (2017). Succinyl-proteome profiling of Dendrobium officinale, an important traditional Chinese orchid herb, revealed involvement of succinylation in the glycolysis pathway. BMC Genomics 18, 598. https://doi.org/ 10.1186/s12864-017-3978-x.

Fernie, A.R., Carrari, F., and Sweetlove, L.J. (2004). Respiratory metabolism: glycolysis, the TCA cycle and mitochondrial electron transport. Curr. Opin. Plant Biol. 7, 254-261. https://doi.org/ 10.1016/j.pbi.2004.03.007.

He, D.L., Wang, Q., Li, M., Damaris, R.N., Yi, X.L., Cheng, Z.Y., and Yan, P.F. (2016). Global proteome analyses of lysine acetylation and succinylation reveal the widespread involvement of both modification in metabolism in the embryo of germinating rice seed. J. Proteome Res. 15, 879-890. https://doi.org/ 10.1021/acs. jproteome.5b00805.

Horii, Y., Tanidaa, M., Shena, J., Hirata, T., Kawamura, N., Wada, A., and Nagai, K. (2010). Effects of Eucommia leaf extracts on autonomic nerves, body temperature, lipolysis, food intake, and body weight. Neurosci. Letters 479, 181-186. https://doi.org/ 10.1016/j. neulet.2010.05.030.

Jin, W.B., and Wu, F.L. (2016). Proteome-wide identification of lysine succinylation in the proteins of tomato (Solanum lycopersicum). PLoS ONE 11, e0147586. https://doi.org/ 10.1371/journal. pone.0147586.

Lara, M.V., Disante, K.B., Podestá, F.E., Andreo, C.S., and Drincovich, M.F. (2003). Induction of a Crassulacean acid like metabolism in the $\mathrm{C}_{4}$ succulent plant, Portulaca oleracea L.: Physiological and morphological changes are accompanied by specific modifications in phosphoenolpyruvate carboxylase. Photosynth. Res. 77, 241-254.

Li, X.L., Hu, X., Wan, Y.J., Xie, G.Z., Li, X.Z., Chen, D., Cheng, Z.Y., Yi, X.L., Liang, S.H., and Tan, F. (2014). Systematic identification of the lysine succinylation in the protozoan parasite Toxoplasma gondii. J. Proteome Res. 13, 6087-6095. https://doi.org/ 10.1021/ pr500992r

Meng, X.X., Mujahid, H., Zhang, Y.D., Peng, X.J., Redoña, E.D., Wang, C.L., and Peng, Z.H. (2019). Comprehensive analysis of the lysine succinylome and protein co-modifications in developing rice seeds. Molec. Cell. Proteomics 18, 2359-2372. https://doi.org/ 10.1074/ mcp.RA119.001426.

Minagawa, J. (2011). State transitions - The molecular remodeling of photosynthetic supercomplexes that controls energy flow in the chloroplast. Biochim. Biophys. Acta 1807, 897-905. https://doi.org/ 10.1016/j.bbabio.2010.11.005.

Mulo, P. (2011). Chloroplast-targeted ferredoxin-NADP+ oxidoreductase (FNR): Structure, function and location. Biochim. Biophys. Acta 1807, 927-934. https://doi.org/ 10.1016/j. bbabio.2010.10.001.

Papanicolaou, K.N., O’Rourke, B., and Foster, D.B. (2014). Metabolism leaves its mark on the powerhouse: Recent progress in posttranslational modifications of lysine in mitochondria. Front. Physiol. 5, 301. https://doi.org/ 10.3389/fphys.2014.00301.

Park, S.A., Choi, M.S., Jung, U.J., Kim, M.J., Kim, D.J., Park, H.M., Park, Y.B., and Lee, M.K. (2006). Eucommia ulmoides Oliver leaf extract increases endogenous antioxidant activity in type 2 diabetic mice. J. Medicinal Food 9, 474-479. https://doi.org/ 10.1089/ jmf.2006.9.474.

Ren, S.L., Yang, M.K., Yue, Y.W., Ge, F., Li, Y., Guo, X.D., Zhang, J., Zhang, F., Nie, X.Y., and Wang, S.H. (2018). Lysine succinylation contributes to Aflatoxin production and pathogenicity in Aspergillus flavus. Molec. Cell. Proteomics 17, 457-471. https://doi.org/ 10.1074/mcp. RA117.000393.

Rosen, R., Becher, D., Büttner, K., Biran, D., Hecker, M., and Ron, E.Z. (2004). Probing the active site of homoserine trans-succinylase. FEBS Letters 577, 386-392. https://doi.org/ 10.1016/j. febslet.2004.10.037. 
Seidler, A. (1996). The extrinsic polypeptides of Photosystem II. Biochim. Biophys. Acta 1277, 35-60. https://doi.org/ 10.1016/ S0005-2728(96)00102-8.

Shen, C.J., Xue, J., Sun, T., Guo, H., Zhang, L., Meng, Y.J., and Wang, H.Z. (2016). Succinyl-proteome profiling of a high taxol containing hybrid Taxus species (Taxus $\times$ media) revealed involvement of succinylation in multiple metabolic pathways. Sci. Rep. 6, 21764. https://doi.org/ 10.1038/srep21764.

Song, Y.X., Wang, J., Cheng, Z.Y., Gao, P., Sun, J.X., Chen, X.W., Chen, C., Wang, Y.L., and Wang, Z.N. (2017). Quantitative global proteome and lysine succinylome analyses provide insights into metabolic regulation and lymph node metastasis in gastric cancer. Sci. Reps. 7 , 42053. https://doi.org/ 10.1038/srep42053.

Spreitzer, R.J., and Salvucci, M.E. (2002). RUBISCO: Structure, regulatory interactions, and possibilities for a better enzyme. Ann. Rev. Plant Biol. 53, 449-475. https://doi.org/ 10.1146/annurev. arplant.53.100301.135233.

Sushabhan, S., Liu, X.J., Ryu, D., Nelsona, O.D., Stupinski, J.A., Li, Z., Chen, W., Zhang, S., Weiss, R.S., Locasale, J.W., Auwerx, J., and Lin, H.N. (2016). Metabolomics-assisted proteomics identifies succinylation and SIRT5 as important regulators of cardiac function. Proc. Nat. Acad. Sci. 113, 4320. https://doi.org/ 10.1073/pnas.1519858113.

Walsh, C.T., Garneau-Tsodikova, S., and Gatto, G.J. (2005). Protein posttranslational modifications: The chemistry of proteome diversifications. Angewandte Chemie, Int. ed. 44, 7342-7372. https://doi.org/ 10.1002/anie.200501023.

Wang, H., Huang, H., Ding, C., and Nie, F. (2013). Predicting proteinprotein interactions from multimodal biological data sources via nonnegative matrix tri-factorization. J. Comp. Biol. 20, 344-358. https://doi.org/ 10.1089/cmb.2012.0273.

Wang, X.B., Wang, X.Z., Li, J.R., Zhou, X.X., Liu, Y.T., Zhong, L.T., Tang, Y., Zheng H., Liu, J.Y., Zhan, R.T., and Chen, L.K. (2019). Global analysis of lysine succinylation in patchouli plant leaves. Hortic. Res. 6, 133. https://doi.org/ 10.1038/s41438-019-0216-5.

Wani, W.Y., Boyer-Guittaut, M., Dodson, M., Chatham, J., DarleyUsmar, V., and Zhang, J.H. (2014). Regulation of autophagy by protein post-translational modification. Lab. Investig. 95, 14-25. https://doi. org/ 10.1038/labinvest.2014.131.

Weinert, B.T., Schölz, C., Wagner, S.A., Iesmantavicius, V., Su, D., Daniel, J.A., and Choudhary, C. (2013). Lysine succinylation is a frequently occurring modification in prokaryotes and eukaryotes and extensively overlaps with acetylation. Cell Rep. 4, 842-851. https://doi.org/ 10.1016/j.celrep.2013.07.024.

Witze, E.S., Old, W.M., Resing, K.A., and Ahn, N.G. (2007). Mapping protein post-translational modifications with mass spectrometry. Nature Meth. 4, 798-806. https://doi.org/ 10.1038/nmeth1100.

Wuyun, T.N., Wang, L., Liu, H.M., Wang, X.W., Zhang, L.S., Bennetzen, J.L., Li, T.Z., Yang, L.R., Liu, P.F., Du, L.Y., et al. (2018). The Hardy rubber tree genome provides insights into the evolution of polyisoprene biosynthesis. Molec. Plant. 11, 429-442. https://doi.org/ 10.1016/j. molp.2017.11.014.

Xie, L.X., Liu, W., Li, Q.M., Chen, S.D., Xu, M.M., Huang, Q.Q., Zeng, J., Zhou, M.L., and Xie, J.Q. (2015). First succinyl-proteome profiling of extensively drug-resistant Mycobacterium tuberculosis revealed involvement of succinylation in cellular physiology. J. Proteome Res. 14, 107-119. https://doi.org/ 10.1021/pr500859a.

Xie, Z.Y., Dai, J.B., Dai, L.Z., Tan, M.J., Cheng, Z.Y., Wu, Y.M., Boeke, J.D., and Zhao, Y.M. (2012). Lysine succinylation and lysine malonylation in histones. Molec. Cell. Proteomics 11, 100-107. https://doi.org/ 10.1074/mcp.M111.015875.
Xu, Y.X., Shen, C.J., Ma, J.Q., Chen, W., Mao, J., Zhou, Y.Y., and Chen, L. (2017). Quantitative succinyl-proteome profiling of Camellia sinensis cv. 'Anji Baicha' during periodic albinism. Sci. Rep. 7, 1873. https:// doi.org/ 10.1038/s41598-017-02128-x.

Yu, Y., Weng, J., and Xu, C.H. (2001). The progress in the investigation on the structure and function of the extrinsic proteins of Photosystem II. Plant Physiol. J. 27, 441-450.

Zhang, Y.M., Song, L.M., Liang, W.X., Mu, P., Wang, S., and Lin, Q. (2016). Comprehensive profiling of lysine acetylproteome analysis reveals diverse functions of lysine acetylation in common wheat. Sci. Rep. 6, 21069. https://doi.org/ 10.1038/srep21069.

Zhang, Y.M., Wang, G.Y., Song, L.M., Mu, P., Wang, S., Liang, W.X., and Lin, Q. (2017). Global analysis of protein lysine succinylation profiles in common wheat. BMC Genomics 18, 309. https://doi.org/ 10.1186/s12864-017-3698-2.

Zhang, Z.H., Tan, M.J., Xie, Z.Y., Dai, L.Z., Chen, Y., and Zhao, T.M. (2010). Identification of lysine succinylation as a new post-translational modification. Nat. Chem. Biol. 7, 58-63. https://doi.org/ 10.1038/ nchembio. 495 .

Zheng, H.L., He, Y., Zhou, X.W., Qian, G.Y., Lv, G.X., Shen, Y.N., Liu, J.Y., Li, D.M., Li, X.F., and Liu, W.D. (2016), Systematic analysis of the lysine succinylome in Candida albicans. J. Proteome Res. 15, 3793-3801. https://doi.org/ 10.1021/acs.jproteome.6b00578.

Zhou, H., Finkemeier, I., Guan, W.X., Tossounian, M.A., Wei, B., Young D., Huang, J.Q., Messens, J., Yang, X.B., Zhu, J., Wilson, M.H., Shen, W.B., Xie, Y.J., and Foyer, C.H. (2017). Oxidative stress-triggered interactions between the succinyl- and acetyl-proteomes of rice leaves. Plant Cell Environm. 41, 1139-1153. https://doi.org/ 10.1111/pce.13100.

Zhu, M.Q., Wen, J.L., Zhua, Y.H., Sua, Y.Q., and Sun, R.C. (2016). Isolation and analysis of four constituents from barks and leaves of Eucommia ulmoides Oliver by a multi-step process. Industr. Crops Prod. 83, 124-132. https://doi.org/ 10.1016/j.indcrop.2015.12.049.

Received: Nov. 25, 2020

Accepted: Jan. 29, 2021

Addresses of authors:

Chao Shen ${ }^{1}$, Xinzhuan Yao ${ }^{1}$, Degang Zhao ${ }^{2}$ and Litang $\mathrm{Lu}^{1,2, *}$

${ }^{1}$ College of Tea Science, Guizhou University, Guiyang, Guizhou, People's Republic of China

${ }^{2}$ The Key Laboratory of Plant Resources Conservation and Germplasm Innovation in Mountainous Region (Ministry of Education), Institute of Agro-Bioengineering, Guiyang, Guizhou, People's Republic of China

*Corresponding author; E-mail: ltlv@gzu.edu.cn 